\title{
A Numerical Convergence Study of some Open Boundary Conditions for Euler Equations
}

\author{
Clément Colas, Martin Ferrand, Jean-Marc Hérard, Olivier Hurisse, Erwan Le \\ Coupanec and Lucie Quibel
}

5 Abstract We discuss herein the suitability of some open boundary conditions. Considering the Euler system of gas dynamics, we compare approximate solutions of one-dimensional Riemann problems in a bounded sub-domain with the restriction in this sub-domain of the exact solution in the infinite domain. Assuming that no information is known from outside of the domain, some basic open boundary con10 dition specifications are given, and a measure of the $L^{1}$-norm of the error inside the computational domain enables to show consistency errors in situations involving outgoing shock waves, depending on the chosen boundary condition formulation. This investigation has been performed with Finite Volume methods, using approximate Riemann solvers in order to compute numerical fluxes for inner interfaces and boundary interfaces.

Key words: Finite volumes, approximate Riemann solver, open boundary conditions, Euler equations, compressible flow

MSC (2010): 65M08, 65N08, 76N15

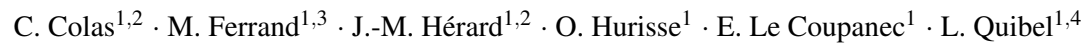
${ }^{1}$ EDF R\&D, MFEE, 6 quai Watier, 78400, Chatou, France

${ }^{2}$ Aix-Marseille Université, I2M, UMR CNRS 7373, 39 rue Joliot Curie, 13453, Marseille, France

${ }^{3}$ CEREA Lab (Ecole des Ponts ParisTech - EDF R\&D), 6-8 avenue Blaise Pascal, Cité Descartes, 77420 Champs-sur-Marne, France

${ }^{4}$ Université de Strasbourg, IRMA, UMR CNRS 7501, 7 rue René Descartes, 67084, Strasbourg,

France

e-mail: clement.colas@edf.fr, martin.ferrand@edf.fr, jean-marc.herard@edf.fr,

olivier.hurisse@edf.fr,erwan.lecoupanec@edf.fr, lucie.quibel@edf.fr
} 


\section{Introduction} be connected to the work of [7]. The solution of Euler system (1) is sought in $\mathbb{R} \times(0, T)$, with time $T \in \mathbb{R}_{+}^{*}$, without boundary conditions, see [14]. This solution, expected to be known and unique, is denoted by $\boldsymbol{W}_{\Omega_{\infty}}^{\text {exact }}(x, t)$ for $(x, t) \in \mathbb{R} \times(0, T)$.

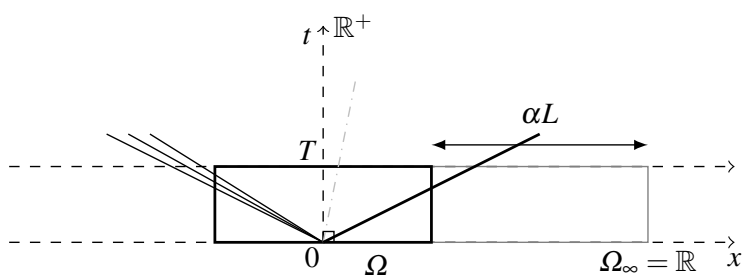

Fig. 1 Bounded computational domain $\Omega \varsubsetneqq \Omega_{\infty}$, with $\Omega_{\infty}$ a spatial infinite domain.

In contrast, the numerical approximations, denoted by $\boldsymbol{W}_{\Omega}^{\Delta x, \Delta t}(x, t)$ for $(x, t) \in$ $\Omega \times(0, T)$, are performed in a bounded computational sub-domain $\Omega \varsubsetneqq \Omega_{\infty}$ (see Fig. 1) with prescribed open inlet/outlet boundary conditions on $\partial \Omega$.

For this purpose, artificial boundaries are introduced on $\partial \Omega$. Then, numerical boundary conditions, depending on the time and space steps, must be prescribed on $\partial \Omega$. When $(\Delta x, \Delta t) \rightarrow(0,0)$, we assume that some (unique) converged approximation, denoted by $\boldsymbol{W}_{\Omega}^{0,0}(x, t)$ for $(x, t) \in \Omega \times(0, T)$, is obtained. Eventually, we wonder whether $\boldsymbol{W}_{\Omega}^{0,0}(x, t)$ for $(x, t) \in \Omega \times(0, T)$, coincides with the restriction of 
the exact solution to $\Omega, W_{\Omega_{\infty}}^{\text {exact }}(x, t)$ for $(x, t) \in \Omega \times(0, T)$, or not. In the latter case, the converged approximation $\boldsymbol{W}_{\Omega}^{0,0}$ will be said to be non-consistent.

For the Euler system (1), a measure of a subsonic state in the last inner cell $N$ (eigenvalues $\lambda_{1}\left(\mathbf{W}_{N}^{n}\right)<0$ and $\lambda_{2,3}\left(\mathbf{W}_{N}^{n}\right)>0$ ) at a right outlet will require one scalar external information, whereas in the supersonic case $\left(\lambda_{1,2,3}\left(\mathbf{W}_{N}^{n}\right)>0\right)$, the upwind state will be privileged. Actually, we recall that in the subsonic case, the approach of $[4,5]$ may provide some way to cope with the lack of information.

A first drawback of the latter approach is that the sign of eigenvalues may easily change: signs of eigenvalues $\lambda_{k}\left(\mathbf{W}_{N}^{n}\right)$ are not necessarily representative of what happens really at the right boundary when computing true waves associated with the 1D Riemann problem with the initial condition: $\boldsymbol{W}_{L}=\mathbf{W}_{N}^{n}$ and $\boldsymbol{W}_{R}=\mathbf{W}_{\text {ext }}^{n}$ (unless when $\mathbf{W}_{e x t}^{n}=\mathbf{W}_{N}^{n}$ ). A very instructive example is given in [7] Sect. 3.2, while restricting on a scalar problem (Burgers equation). A second question is: assuming that nothing is known about the exterior state $\mathbf{W}_{\text {ext }}^{n}$, how does the solution, inside the computational sub-domain, depend on the choice of $\mathbf{W}_{e x t}^{n}$ ?

Herein, the aim consists in testing suitable numerical boundary conditions in the sense that they converge towards the - not necessarily regular - exact solution.

\section{Finite volume method}

We briefly recall the basis of the explicit finite volume scheme VFRoe-ncv, an approximate Godunov scheme using non conservative variables [9, 8]. For the sake of simplicity, regular meshes of the one-dimensional computational domain are considered of size $\Delta x=x_{i+1 / 2}-x_{i-1 / 2}, i \in\{1, \ldots, N\}$, and $\Delta t^{n}=t^{n+1}-t^{n}$ is the time step, $n \in \mathbb{N}$. The time step is given by some CFL condition in order to gain stability. Let $\mathbf{W}_{i}^{n}$ be an approximation of the mean value $\frac{1}{\Delta x} \int_{x_{i-1 / 2}}^{x_{i+1 / 2}} \mathbf{W}\left(x, t^{n}\right) d x$. Time-space integration of system (1) over $\left[x_{i-1 / 2}, x_{i+1 / 2}\right] \times\left[t^{n}, t^{n+1}\right]$ provides the standard following scheme:

$$
\Delta x\left(\mathbf{W}_{i}^{n+1}-\mathbf{W}_{i}^{n}\right)+\Delta t^{n}\left(\mathbf{g}_{i+\frac{1}{2}}^{n}-\mathbf{g}_{i-\frac{1}{2}}^{n}\right)=0
$$

where $\mathbf{g}_{i+1 / 2}^{n}$ is the numerical flux through the interface $\left\{x_{i+1 / 2}\right\} \times\left[t^{n}, t^{n+1}\right]$. For so-called spatially first-order scheme, $\mathbf{g}_{i+1 / 2}^{n}=\mathbf{g}\left(\mathbf{W}_{i}^{n}, \mathbf{W}_{i+1}^{n}\right)$. The numerical flux $\mathbf{g}_{i+1 / 2}^{n}$ is obtained by solving the linearized Riemann problem:

$$
\left\{\begin{array}{l}
\partial_{t} \boldsymbol{Y}+\boldsymbol{B}(\tilde{\boldsymbol{Y}}) \partial_{x} \boldsymbol{Y}=0 \\
\boldsymbol{Y}\left(\boldsymbol{x}, t^{n}\right)= \begin{cases}\mathbf{Y}_{i}^{n} & \text { if } x<x_{i+\frac{1}{2}} \\
\mathbf{Y}_{i+1}^{n} & \text { if } x>x_{i+\frac{1}{2}}\end{cases}
\end{array}\right.
$$

where $\widetilde{\boldsymbol{Y}}=\left(\mathbf{Y}_{i}^{n}+\mathbf{Y}_{i+1}^{n}\right) / 2$ and $\boldsymbol{B}(\boldsymbol{Y})$ stands for the following matrix: 


$$
\boldsymbol{B}(\boldsymbol{Y})=\left(\partial_{\boldsymbol{Y}} \boldsymbol{W}\right)^{-1} \partial_{\boldsymbol{W}} \boldsymbol{F}(\boldsymbol{W}) \partial_{\boldsymbol{Y}} \boldsymbol{W} .
$$

Once the exact solution $\boldsymbol{Y}^{\star}\left(\frac{x-x_{i+1 / 2}}{t} ; \mathbf{Y}_{i}^{n}, \mathbf{Y}_{i+1}^{n}\right)$ of problem (3) is computed, the numerical flux is defined as:

$$
\mathbf{g}_{i+\frac{1}{2}}^{n}=\mathbf{g}\left(\mathbf{W}_{i}^{n}, \mathbf{W}_{i+1}^{n}\right)=\boldsymbol{F}\left(\boldsymbol{W}\left(\boldsymbol{Y}^{\star}\left(0 ; \mathbf{Y}_{i}^{n}, \mathbf{Y}_{i+1}^{n}\right)\right) .\right.
$$

This numerical flux will be used for both inner interfaces and boundary interfaces.

\section{Numerical boundary conditions for outgoing waves}

We propose numerical artificial boundary conditions when no information is given on the open boundary of the computational sub-domain. One possible approach is to determine an artificial state $\mathbf{W}_{\text {ext }}^{n}$ in the virtual cell, symmetric of the boundary cell $\mathbf{W}_{i}^{n}$, outside of the sub-domain. The numerical boundary flux is then obtained by $\mathbf{g}_{1 / 2}^{n}=\mathbf{g}\left(\mathbf{W}_{e x t, 1}^{n}, \mathbf{W}_{1}^{n}\right)$ and $\mathbf{g}_{N+1 / 2}^{n}=\mathbf{g}\left(\mathbf{W}_{N}^{n}, \mathbf{W}_{e x t, N}^{n}\right)$. In the following, we assume

85 that the exterior state is connected to the interior state either by a rarefaction wave or a shock wave.

\subsection{Outgoing rarefaction wave}

a. Formulation assuming the invariance of the interior state $\mathrm{BC}_{0}$

The first boundary condition, widely used in industrial simulations, simply consists in taking the interior state $\mathbf{W}_{i}^{n}$ of the boundary cell at each time step $t^{n}$

$$
\mathbf{W}_{\text {ext }}^{n}=\mathbf{W}_{N}^{n} \text {. }
$$

The numerical boundary flux thus reads $\mathbf{g}_{N+1 / 2}^{n}=\mathbf{g}\left(\mathbf{W}_{N}^{n}, \mathbf{W}_{N}^{n}\right)=\boldsymbol{F}\left(\mathbf{W}_{N}^{n}\right)$. This technique does not need any knowledge about the wave structure.

b. Formulation using the wave structure and an extrapolation of the interior state $\mathrm{BC}_{\mathrm{r}}$

The second boundary condition is built by using the two associated Riemann invariants of the regular wave and a third additional scalar relation. Note that, for an ideal gas, the exact velocity profile is linear w.r.t. $x$ at time $t^{n}$. Thus, for an ideal gas EOS such that $\rho \varepsilon=P /(\gamma-1)$, with $\gamma>1$, we get:

$$
\rho_{e x t}^{n}=\rho_{N}^{n}\left(1-\frac{\gamma-1}{2} \frac{u_{N-1}^{n}-u_{N}^{n}}{c_{N}^{n}}\right)^{\frac{2}{\gamma-1}}, P_{e x t}^{n}=P_{N}^{n}\left(1-\frac{\gamma-1}{2} \frac{u_{N-1}^{n}-u_{N}^{n}}{c_{N}^{n}}\right)^{\frac{2 \gamma}{\gamma-1}}
$$

and $u_{e x t}^{n}=2 u_{N}^{n}-u_{N-1}^{n}$. The numerical boundary flux is computed by $\mathbf{g}_{N+1 / 2}^{n}=$ $\mathbf{g}\left(\mathbf{W}_{N}^{n}, \mathbf{W}_{e x t}^{n}\right)$. This technique connects the interior state with the exterior virtual state by using the rarefaction wave structure. 


\subsection{Outgoing shock wave}

c. Formulation assuming the invariance of the interior state $\mathrm{BC}_{0}$

Same as for rarefaction wave, see case $a$. (5).

\section{Numerical results}

We discuss below some results of this preliminary study. Other results with distinct EOS are available in [2]. Two subsonic test cases, corresponding to 1D Riemann problems with a diatomic ideal gas $\operatorname{EOS}\left(\gamma=\frac{7}{5}\right)$, are performed with $\mathrm{CFL}=0.5$. The first one is a pure left outgoing 1-rarefaction wave with the initial condition:

$$
\left\{\begin{array}{l}
\left(\rho_{L}, u_{L}, P_{L}\right)=\left(1 \mathrm{~kg} / \mathrm{m}^{3}, 0 \mathrm{~m} / \mathrm{s}, 10^{5} \mathrm{~Pa}\right) \\
\left(\rho_{R}, u_{R}, P_{R}\right)=\left(0.5 \mathrm{~kg} / \mathrm{m}^{3}, 242.2 \mathrm{~m} / \mathrm{s}, 3.789 \times 10^{4} \mathrm{~Pa}\right) .
\end{array}\right.
$$

The second one is a pure right outgoing 3-shock wave with the initial condition:

$$
\left\{\begin{array}{l}
\left(\rho_{L}, u_{L}, P_{L}\right)=\left(1 \mathrm{~kg} / \mathrm{m}^{3}, 418.3 \mathrm{~m} / \mathrm{s}, 2.75 \times 10^{5} \mathrm{~Pa}\right), \\
\left(\rho_{R}, u_{R}, P_{R}\right)=\left(0.5 \mathrm{~kg} / \mathrm{m}^{3}, 0 \mathrm{~m} / \mathrm{s}, 10^{5} \mathrm{~Pa}\right) .
\end{array}\right.
$$

The numerical convergence of the scheme, when waves are gone out of the bounded computational domain $\Omega=(-200 \mathrm{~m}, 200 \mathrm{~m})$, is measured with the $L^{1}$-norm of the error.

For smooth waves, the boundary conditions $\mathrm{BC}_{0}$ and $\mathrm{BC}_{\mathrm{r}}$ enable to guarantee consistency when waves are going out $\left(t_{0}<t<t_{1}\right)$ or are gone out $\left(t>t_{1}\right)$ of $\Omega$. The numerical errors and the rates of convergence are collected in Table 1 and Fig. 2 for an outgoing rarefaction wave, and in Table 2 and Fig. 3 when the whole rarefaction wave has left the computational domain. As expected for an ideal gas EOS [8], the numerical rates of convergence for variables $(u, P)$ are approximately $0.85-$ close 
to $1-$ when $t<t_{1}$ (see Table 1), and thus similar to those arising for $t<t_{0}$, see $[8,9]$. Table 2 shows greater orders of convergence which may be due to the fact that the exact solution becomes fully constant for $t>t_{1}$. The $\mathrm{BC}_{\mathrm{r}}$ condition gives very similar errors and does not provide more accurate approximations.

In contrast, the $\mathrm{BC}_{0}$ condition does not ensure the consistency of the scheme for an outgoing shock wave (at $t>t_{0}$, shock is outside of $\Omega$ ), see Fig. 4: clearly, approximate solutions converge towards another solution when $(\Delta x, \Delta t) \rightarrow(0,0)$.

The $\mathrm{BC}_{\mathrm{s}}$ boundary condition, for a finite value of the parameter $\alpha>0$, is still not consistent, see Fig. 5. At the limit $\alpha \rightarrow+\infty$, the asymptotic condition $\mathrm{BC}_{\mathrm{s}}$ allows to retrieve the consistency of the approximate solution with the exact solution.

Further works aim at considering another boundary condition for outgoing shock waves based on an imposed scalar value outside and the Rankine-Hugoniot relations. The issue of the supersonic shock wave case and of the dependence on the scheme [13] are being examined. To our knowledge, this measured loss of consistency has not been pointed out before.

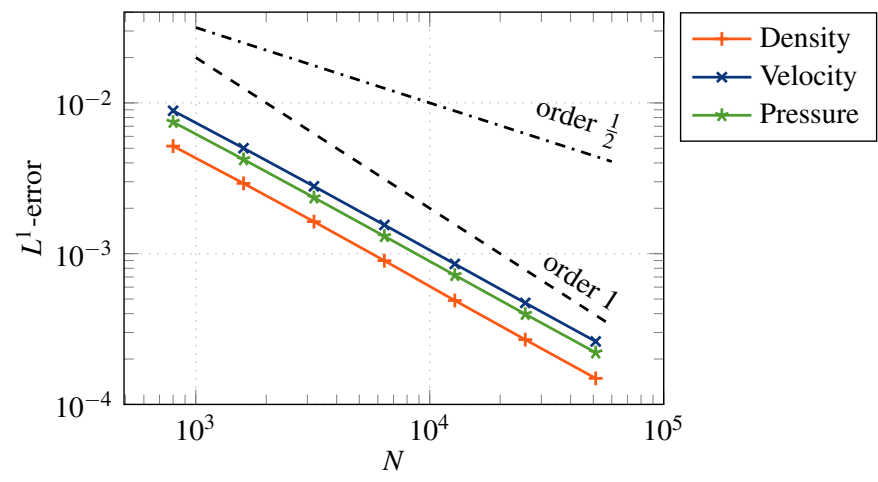

Fig. $2 \mathrm{BC}_{0}: L^{1}$ convergence curves for the rarefaction wave at $t_{0}<t<t_{1}$.

Table $1 \mathrm{BC}_{0}: L^{1}$ convergence orders for the rarefaction wave at $t_{0}<t<t_{1}$.

\begin{tabular}{llllllll}
\hline$\Delta x(\mathrm{~m})$ & $N$ & $\rho L^{1}$-error & $\rho$ cnv. order & $u L^{1}$-error & $u$ cnv. order & $P L^{1}$-error & $P$ cnv. order \\
\hline $5 \mathrm{e}-1$ & 800 & $5.172 \mathrm{e}-3$ & & $8.868 \mathrm{e}-3$ & & $2.371 \mathrm{e}-3$ & \\
$2.5 \mathrm{e}-1$ & 1600 & $2.925 \mathrm{e}-3$ & 0.8221 & $5.009 \mathrm{e}-3$ & 0.8241 & $1.335 \mathrm{e}-3$ & 0.8243 \\
$1.25 \mathrm{e}-1$ & 3200 & $1.631 \mathrm{e}-3$ & 0.8426 & $2.798 \mathrm{e}-3$ & 0.8403 & $7.478 \mathrm{e}-4$ & 0.8402 \\
$6.25 \mathrm{e}-2$ & 6400 & $8.984 \mathrm{e}-4$ & 0.8605 & $1.550 \mathrm{e}-3$ & 0.8518 & $4.194 \mathrm{e}-4$ & 0.8516 \\
$3.125 \mathrm{e}-2$ & 12800 & $4.891 \mathrm{e}-4$ & 0.8774 & $8.548 \mathrm{e}-4$ & 0.8587 & $2.379 \mathrm{e}-4$ & 0.8582 \\
$1.5625 \mathrm{e}-2$ & 25600 & $2.691 \mathrm{e}-4$ & 0.8621 & $4.714 \mathrm{e}-4$ & 0.8588 & $1.386 \mathrm{e}-4$ & 0.8579 \\
$7.8125 \mathrm{e}-3$ & 51200 & $1.489 \mathrm{e}-4$ & 0.8533 & $2.617 \mathrm{e}-4$ & 0.8491 & $8.461 \mathrm{e}-5$ & 0.8474 \\
\hline
\end{tabular}




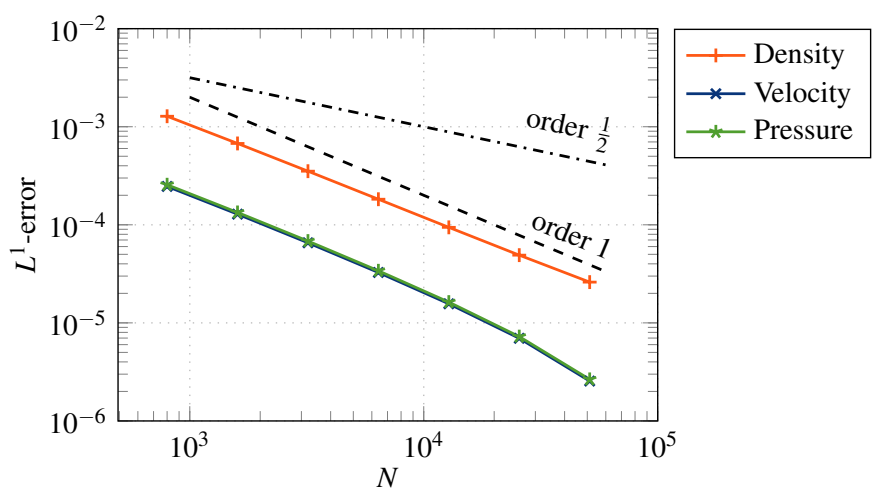

Fig. $3 \mathrm{BC}_{0}: L^{1}$ convergence curves for the rarefaction wave at $t>t_{1}$.

Table $2 \mathrm{BC}_{0}: L^{1}$ convergence orders for the rarefaction wave at $t>t_{1}$.

\begin{tabular}{llllllll}
\hline$\Delta x(\mathrm{~m})$ & $N$ & $\rho L^{1}$-error & $\rho$ cnv. order & $u L^{1}$-error & $u$ cnv. order & $P L^{1}$-error & $P$ cnv. order \\
\hline $5 \mathrm{e}-1$ & 800 & $1.279 \mathrm{e}-3$ & & $2.462 \mathrm{e}-4$ & & $2.562 \mathrm{e}-4$ & \\
$2.5 \mathrm{e}-1$ & 1600 & $6.755 \mathrm{e}-4$ & 0.9211 & $1.284 \mathrm{e}-4$ & 0.9384 & $1.337 \mathrm{e}-4$ & 0.9383 \\
$1.25 \mathrm{e}-1$ & 3200 & $3.522 \mathrm{e}-4$ & 0.9395 & $6.557 \mathrm{e}-5$ & 0.9700 & $6.826 \mathrm{e}-5$ & 0.9700 \\
$6.25 \mathrm{e}-2$ & 6400 & $1.823 \mathrm{e}-4$ & 0.9502 & $3.265 \mathrm{e}-5$ & 1.0061 & $3.399 \mathrm{e}-5$ & 1.0061 \\
$3.125 \mathrm{e}-2$ & 12800 & $9.423 \mathrm{e}-5$ & 0.9521 & $1.565 \mathrm{e}-5$ & 1.0608 & $1.629 \mathrm{e}-5$ & 1.0609 \\
$1.5625 \mathrm{e}-2$ & 25600 & $4.904 \mathrm{e}-5$ & 0.9420 & $6.962 \mathrm{e}-6$ & 1.1687 & $7.247 \mathrm{e}-6$ & 1.1687 \\
$7.8125 \mathrm{e}-3$ & 51200 & $2.604 \mathrm{e}-5$ & 0.9134 & $2.551 \mathrm{e}-6$ & 1.4486 & $2.655 \mathrm{e}-6$ & 1.4486 \\
\hline
\end{tabular}

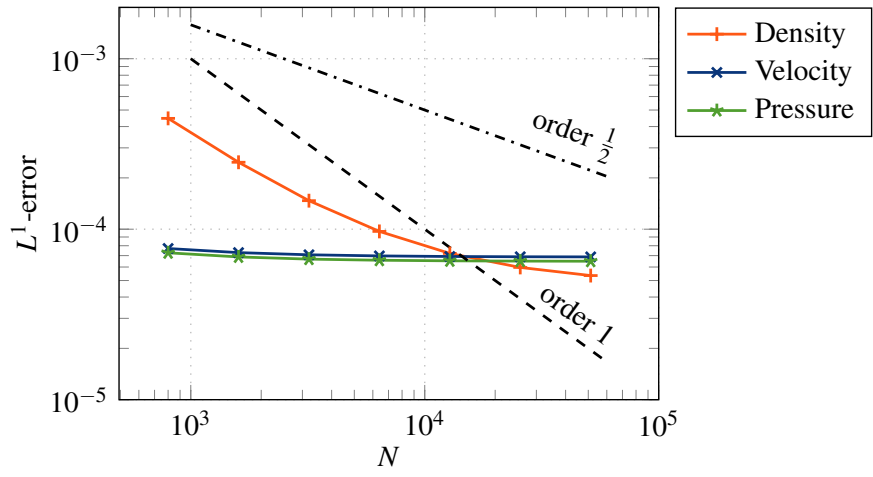

Fig. $4 \mathrm{BC}_{0}: L^{1}$ convergence curves for the shock wave at $t>t_{0}$.

Acknowledgements The first author received a financial support by ANRT through an EDFCIFRE contract 2016/0728. The last author also receives a financial support by ANRT through an EDF-CIFRE contract 2017/0476. All computational facilities were provided by EDF R\&D. 


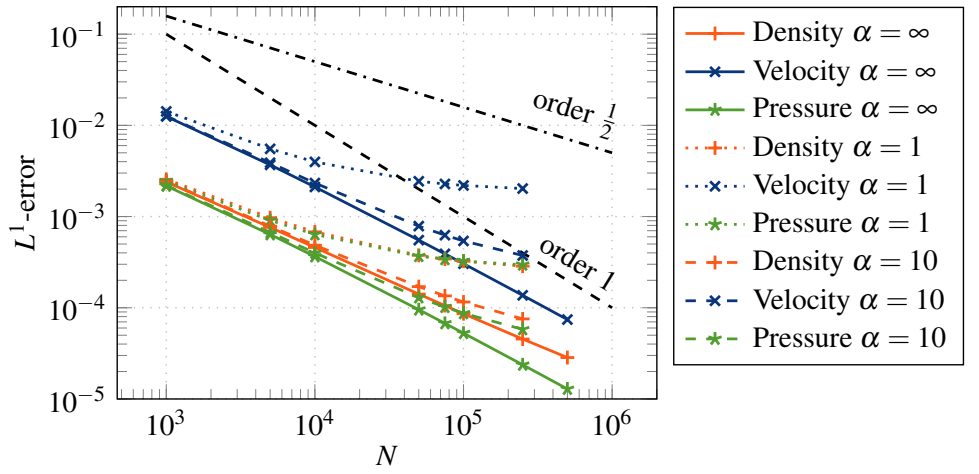

Fig. $5 \mathrm{BC}_{\mathrm{s}}: L^{1}$ convergence curves for the shock tube at $t>t_{0}$.

\section{References}

1. Bardos, C., LeRoux, A.Y., Nédélec, J.C.: First order quasilinear equations with boundary conditions. Communications in Partial Differential Equations 4(9), 1017-1034 (1979)

2. Colas, C.: Time-implicit integral formulation for fluid flow modelling in congested media. PhD thesis, Aix-Marseille Université (2019). URL https://tel.archives-ouvertes.fr/tel02382958

3. Deininger, M., Iben, U., Munz, C.D.: Coupling of three- and one-dimensional hydraulic flow simulations. Computers \& Fluids 190, 128 - 138 (2019)

4. Dubois, F.: Boundary conditions and the Osher scheme for the Euler equations of gas dynamics. Internal Report CMAP 170, Ecole Polytechnique, Palaiseau, France (1987)

5. Dubois, F., Le Floch, P.: Boundary conditions for nonlinear hyperbolic systems of conservation laws. J. Diff Equations 71(1), 93-122 (1988)

6. Engquist, B., Majda, A.: Absorbing boundary conditions for the numerical simulation of waves. Mathematics of Computation 31(139), 629-651 (1977)

7. Gallouët, T.: Boundary conditions for hyperbolic equations or systems. In: M. Feistauer, V. Dolejší, P. Knobloch, K. Najzar (eds.) Numerical Mathematics and Advanced Applications, pp. 39-55. Springer Berlin Heidelberg, Berlin, Heidelberg (2004)

8. Gallouët, T., Hérard, J.M., Seguin, N.: Some recent Finite Volume schemes to compute Euler equations using real gas EOS. International Journal for Numerical Methods in Fluids 39, 1073-1138 (2002)

9. Gallouët, T., Hérard, J.M., Seguin, N.: On the use of symmetrizing variables for vacuum. Calcolo 40(3), 163-194 (2003)

10. Hedstrom, G.W.: Nonreflecting boundary conditions for nonlinear hyperbolic systems. Journal of Computational Physics 30, 222-237 (1979)

11. Orlanski, I.: A simple boundary condition for unbounded hyperbolic flows. Journal of Computational Physics 21(3), 251-269 (1976)

12. Poinsot, T.J., Lele, S.K.: Boundary conditions for direct simulations of compressible viscous flows. Journal of Computational Physics 101(1), 104-129 (1992)

13. Quibel, L.: Simulation d'écoulements diphasiques eau-vapeur avec un modèle homogène. $\mathrm{PhD}$ thesis in preparation, Université de Strasbourg. URL http://www.theses.fr/s188859

14. Smoller, J.: Shock Waves and Reaction-Diffusion Equations, A Series of Comprehensive Studies in Mathematics, vol. 258. Springer-Verlag, New York (1994) 